\title{
Relevance of many-body interactions for correlated electrons in the strong-coupling limit
}

\author{
T.R. Kirkpatrick \\ Institute for Physical Science and Technology, and Department of Physics \\ University of Maryland, College Park, MD 20742 \\ D.Belitz \\ Department of Physics and Materials Science Institute, University of Oregon, Eugene, OR 97403
}

(Dated: November 23, 2018)

\begin{abstract}
Many-body interactions in effective field theories for disordered interacting electrons are considered. It is shown that three-body and higher interaction terms are generated in perturbation theory, and some of the physical consequences of these interactions are discussed. It is shown in particular that they will in general be important for any effects governed by strong-coupling fixed points. This implies that the usual generalized nonlinear sigma-model for disordered electron systems is incomplete, and not suitable for studying strong-coupling effects.
\end{abstract}

PACS numbers: 71.10.-w; 71.27.+a; 71.30.+h

\section{INTRODUCTION}

Effective field theories are a very useful tool, both in statistical mechanics and in particle physics $\underline{1}$ The basic idea is to construct an "effective" theory, valid at large length scales and long times, which contains only those degrees that are important in this regime, while all others have been integrated out. Since the effective theory is simpler than the underlying fundamental theory that contains all degrees of freedom explicitly, it is easier to solve. If the fundamental theory is known, as is the case in condensed matter physics, effective theories can be derived from it. If it is not known, as in particle physics, the effective theory can be guessed with feedback from experiments. In either case the effective theory in general contains features that are not present in the fundamental or microscopic theory. For instance, interaction events between particles that are sequential occurences of fundamental interactions on microscopic scales will appear as basic interactions on the coarse-grained length and time scales of the effective theory. An example from particle physics is the Fermi theory of beta decay, which assumed a point-like interaction between the particles involved ${ }^{2}$ Later, in more microscopic theories of the weak interaction, it became clear that there is internal structure in Fermi's interaction related to the exchange of gauge bosons $\frac{3}{\underline{3}}$

In condensed matter physics, the only interaction in the microscopic theory is the Coulomb interaction. We will be concerned with electron-electron interactions in disordered metals, and therefore we take the "fundamental" interaction to be the screened Coulomb interaction. Let us consider processes in which two electrons interact at some point in space and time, and some time later a third electron interacts with one of the two some distance away from the first interaction point. In an effective theory that has integrated out the behavior at short length and time scales, such a process will appear as a "fundamental" interaction between three electrons, since the effective theory can no longer resolve the individual microscopic interaction processes. In classical statistical mechanics the importance of such effective many-body interactions is well known. An example is the expansion of transport coefficients in powers of the particle number density. To obtain the contribution at any given (sufficiently high) order in the density one needs to consider collisions between arbitrarily many particles. $\stackrel{4}{*}$ Analogous effects have been considered for many-electron systems,,$\frac{5}{,}$ although the connection with effective many-body interactions was not made explicit. Furthermore, the construction of a complete effective theory requires that any many-body interactions that are generated in perturbation theory be included in further iterations of the renormalization process that integrates out the short-range degrees of freedom. This has never been done; existing effective theories for disordered many-electron systems contain two-body interactions only $\underline{\underline{6}}$

In the present paper, we show explicitly that manybody interactions in such systems are generated under renormalization. The many-body interactions generated are of long range in space and time due to the diffusive electron dynamics. As a consequence of their long-range nature, the naive renormalization-group (RG) scale dimensions of these terms vanish in two-dimensions, which implies that they should be important in theories of the Anderson-Mott metal-insulator transition near twodimensions ${ }^{6}$ We will clarify in what sense this is the case. We will further show that these many-body interactions can lead to qualitatively new scaling behavior if the interactions are strong enough.

The organization of this paper is as follows. In Sec. III we give simple physical arguments for the existence of effective many-body interactions and their expected structure. In Sec. III we perform an explicit calculation showing that such terms are indeed generated in perturbation theory, starting with a model that has two-body interactions only. In Sec. IV we discuss the physical con- 
sequences of these terms, and in particular their relevance for strong-coupling problems. Section $\nabla$ contains a conclusion, and in the appendix we discuss some aspects of $\phi^{4}$-theory that are analogous in some respects to our perturbative calculation.

\section{PHYSICAL ARGUMENTS FOR THE EXISTENCE OF EFFECTIVE MANY-BODY INTERACTIONS}

Let us consider an ensemble of interacting electrons in the presence of quenched disorder. For simplicity, we will model the screened Coulomb interaction by an instantaneous, point-like model interaction whose coupling constant we denote by $K^{(2)}$. The action will therefore contain a term

$$
\begin{aligned}
S_{\mathrm{int}}^{(2)} & =K^{(2)} \int d \boldsymbol{x} d \boldsymbol{y} \int_{0}^{\beta} d \tau n(\boldsymbol{x}, \tau) \delta(\boldsymbol{x}-\boldsymbol{y}) n(\boldsymbol{y}, \tau) \\
& =K^{(2)} \int d \boldsymbol{x} d \boldsymbol{y} T \sum_{n} n\left(\boldsymbol{x}, \Omega_{n}\right) \delta(\boldsymbol{x}-\boldsymbol{y}) n\left(\boldsymbol{y},-\Omega_{n}\right) .
\end{aligned}
$$

Here $n$ is the electron number density field, which is a function of position $\boldsymbol{x}$ and imaginary time $\tau$, and $\beta=1 / T$ is the inverse temperature. We use units such that $\hbar=k_{\mathrm{B}}=1$. In the second line we have performed a Fourier transform from imaginary time to bosonic Matubara frequencies $\Omega_{n}=2 \pi T n$.

If neither the disorder nor the interaction is too

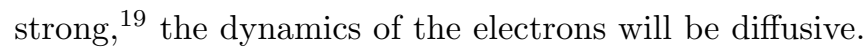
This means there are particle-hole excitations, or diffusons, that are described by a diffusive propagator

$$
\mathcal{D}_{n}(\boldsymbol{x}-\boldsymbol{y})=\delta(\boldsymbol{x}-\boldsymbol{y})\left(-D \nabla^{2}+\left|\Omega_{n}\right|\right)^{-1} .
$$

The exchange of diffusons then provides an effective longranged interaction between the electrons. Consider, for instance, three electrons that are pairwise coupled by diffusion propagators, see Fig. 10 Each two-body interaction carries an amplitude $K^{(2)}$, and we therefore expect this

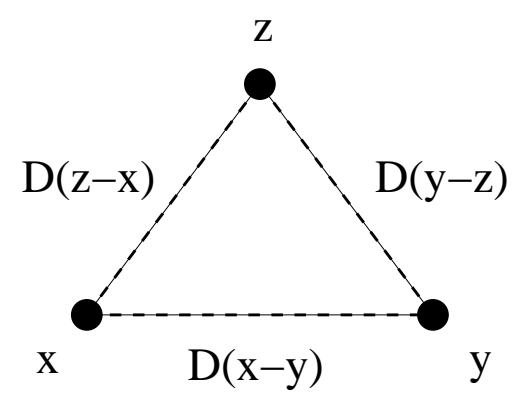

FIG. 1: An effective three-body interaction mediated by three diffusion propagators.

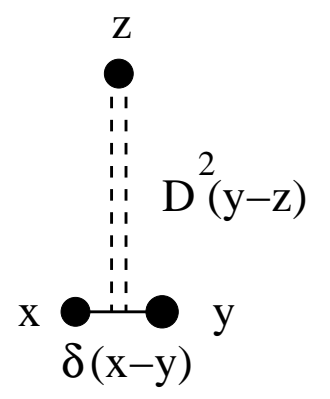

FIG. 2: An effective three-body interaction mediated by two diffusion propagators.

process to contribute a term to the effective action,

$$
\begin{aligned}
S_{\text {int }}^{(3,1)}= & \left(K^{(2)}\right)^{3} \int d \boldsymbol{x} d \boldsymbol{y} d \boldsymbol{z} \int_{0}^{\beta} d \tau T \sum_{m} n(\boldsymbol{x}, \tau) \\
& \times \mathcal{D}_{m}(\boldsymbol{x}-\boldsymbol{y}) n(\boldsymbol{y}, \tau) \mathcal{D}_{m}(\boldsymbol{y}-\boldsymbol{z}) n(\boldsymbol{z}, \tau) \\
& \times \mathcal{D}_{m}(\boldsymbol{z}-\boldsymbol{x}) .
\end{aligned}
$$

Here we have localized the diffusons in imaginary time, i.e., we have neglected their dependence on the external frequency arguments. This implies an effective threeelectron interaction amplitude, defined in analogy to Eq. (2.1), that is given by

$$
\begin{array}{rl}
K^{(3,1)}(\boldsymbol{x}, \boldsymbol{y}, \boldsymbol{z})=\left(K^{(2)}\right)^{3} & T \sum_{m} \mathcal{D}_{m}(\boldsymbol{x}-\boldsymbol{y}) \mathcal{D}_{m}(\boldsymbol{y}-\boldsymbol{z}) \\
& \times \mathcal{D}_{m}(\boldsymbol{z}-\boldsymbol{x})
\end{array}
$$

In an effective theory that cannot resolve the positions $\boldsymbol{x}$, $\boldsymbol{y}$, and $\boldsymbol{z}, K^{(3,1)}$ will appear as a point-like three-electron interaction. For later referece we note that in momentum space, and with the external momenta put equal to zero, $K^{(3,1)}$ reads

$$
K^{(3,1)}=\left(K^{(2)}\right)^{3} T \sum_{m} \frac{1}{V} \sum_{\mathbf{k}}\left(\mathcal{D}_{m}(\mathbf{k})\right)^{3} .
$$

Notice that the frequency-momentum integral in this expression is infrared divergent in all spatial dimensions $d \leq 4$. This singularity will be cut off by any nonzero external momenta and frequencies. Physically, this means that the three-body interaction is of long range in space and time, as was mentioned in the Introduction. We will come back to this point in Secs. III] and IV below.

While $K^{(3,1)}$ is perhaps the most obvious three-body interaction term, it is easy to see that there are others, including terms that are only quadratic in the two-body interaction amplitude $K^{(2)}$. Consider the situation in Fig. 2] where two electrons coupled by the original shortranged two-body interaction interact with a third one by exchanging diffusons. Since there are two electrons at the same point in space in this process, we expect the long-ranged effective interaction to be mediated by a 
diffuson squared. This leads to an effective three-electron interaction amplitude, at zero external frequencies,

$$
K^{(3,2)}(\boldsymbol{x}, \boldsymbol{y}, \boldsymbol{z})=\left(K^{(2)}\right)^{2} \delta(\boldsymbol{x}-\boldsymbol{y})\left(\mathcal{D}_{n=0}(\boldsymbol{y}-\boldsymbol{z})\right)^{2} .
$$

It is obvious that there cannot be any three-electron interaction terms that are linear in $K^{(2)}$.

Analogous arguments lead to the conclusion that there are four-electron interaction terms, starting at order $\left(K^{(2)}\right)^{3}$, etc. In the following section we will ascertain the existence of such many-body interactions by means of an explicit perturbative calculation for a specific model.

\section{GENERATION OF MANY-BODY INTERACTIONS IN PERTURBATION THEORY}

\section{A. Generalized nonlinear sigma-model}

We now turn to an explicit calculation that shows how many-body interactions are generated by renormalizing models that contain two-body interactions only. For definiteness, we take as our starting point the generalized nonlinear sigma-model for disordered, interacting electrons ${ }^{7}$ that has been used extensively to describe metal-insulator transitions,$\frac{6.8 .9}{,}$ as well as magnetic transitions ${ }^{6}$ in solids. The action reads,

$$
\begin{aligned}
\mathcal{A}= & \frac{-1}{2 G} \int d \boldsymbol{x} \operatorname{tr}(\boldsymbol{\nabla} \hat{Q}(\boldsymbol{x}))^{2}+2 H \int d \boldsymbol{x} \operatorname{tr}(\Omega \hat{Q}(\boldsymbol{x})) \\
& +\mathcal{A}_{\text {int }}^{(2)}[\hat{Q}] .
\end{aligned}
$$

Here $Q$ is a hermitian matrix field subject to the constraints

$$
\hat{Q}^{2}(\boldsymbol{x})=1 \quad, \quad \operatorname{tr} \hat{Q}(\boldsymbol{x})=0 \quad .
$$

The matrix elements $Q_{n m}^{\alpha \beta}$ carry fermionic Matsubara frequency labels $n, m$, and replica labels $\alpha$ to deal with the quenched disorder. The matrix elements are themselves four-by-four matrices to allow for spin and particle-hole degrees of freedom. They constitute the soft-mode components of an underlying matrix field $Q$ that comprises bilinear products of fermionic fields $\bar{\psi}$ and $\psi$ according to the correspondence

$$
Q_{12} \cong \frac{i}{2}\left(\begin{array}{rrrr}
-\psi_{1 \uparrow} \bar{\psi}_{2 \uparrow} & -\psi_{1 \uparrow} \bar{\psi}_{2 \downarrow} & -\psi_{1 \uparrow} \psi_{2 \downarrow} & \psi_{1 \uparrow} \psi_{2 \uparrow} \\
-\psi_{1 \downarrow} \bar{\psi}_{2 \uparrow} & -\psi_{1 \downarrow} \bar{\psi}_{2 \downarrow} & -\psi_{1 \downarrow} \psi_{2 \downarrow} & \psi_{1 \downarrow} \psi_{2 \uparrow} \\
\bar{\psi}_{1 \downarrow} \bar{\psi}_{2 \uparrow} & \bar{\psi}_{1 \downarrow} \bar{\psi}_{2 \downarrow} & \bar{\psi}_{1 \downarrow} \psi_{2 \downarrow} & -\bar{\psi}_{1 \downarrow} \psi_{2 \uparrow} \\
-\bar{\psi}_{1 \uparrow} \bar{\psi}_{2 \uparrow} & -\bar{\psi}_{1 \uparrow} \bar{\psi}_{2 \downarrow} & -\bar{\psi}_{1 \uparrow} \psi_{2 \downarrow} & \bar{\psi}_{1 \uparrow} \psi_{2 \uparrow}
\end{array}\right) .
$$

Here all fields are understood to be taken at position $\boldsymbol{x}$, and $1 \equiv\left(n_{1}, \alpha_{1}\right)$, etc., comprises both frequency and replica labels. It is convenient to expand the $4 \times 4$ matrices in a spin-quaternion basis,

$$
\hat{Q}_{12}(\mathbf{x})=\sum_{r, i=0}^{3}\left(\tau_{r} \otimes s_{i}\right)_{r}^{i} \hat{Q}_{12}(\mathbf{x}) .
$$

Here $\tau_{0}=s_{0}=\mathbb{1}_{2}$ is the $2 \times 2$ unit matrix, and $\tau_{j}=-s_{j}=-i \sigma_{j},(j=1,2,3)$, with $\sigma_{1,2,3}$ the Pauli matrices. In this basis, $i=0$ and $i=1,2,3$ describe the spin-singlet and the spin-triplet, respectively. An explicit calculation reveals that $r=0,3$ corresponds to the particle-hole channel (i.e., products $\bar{\psi} \psi$ ), while $r=1,2$ describes the particle-particle channel (i.e., products $\bar{\psi} \bar{\psi}$ or $\psi \psi$ ). In this basis, the electron number density field as a function of $\boldsymbol{x}$ and a bosonic Matsubara frequency $\Omega_{n}$ is represented by

$$
n\left(\mathbf{x}, \Omega_{n}\right)=\sum_{r=0,3}(\sqrt{-1})^{r} \sum_{m} \operatorname{tr}\left(\tau_{r} \otimes s_{0}\right) Q_{m, m+n}(\boldsymbol{x})
$$

$G$ and $H$ in Eq. (3.1a) are coupling constants that represent the disorder strength and the frequency coupling, respectively. Their bare values are proportional to the resistivity in Boltzmann approximation, and to the density of states in self-consistent Born approximation, respectively. $\Omega$ is a frequency matrix with matrix elements

$$
\Omega_{12}=\left(\tau_{0} \otimes s_{0}\right) \delta_{12} 2 \pi T\left(n_{1}+1 / 2\right),
$$

The final term in Eq. (3.1a) describes the two-body electron-electron interaction. From Eqs. (3.23.4 it is clear that it must be quadratic in $\hat{Q}$. If one separates the interaction into a spin-singlet interaction between number densities, and a spin-triplet interaction between spin densities, $\mathcal{A}_{\text {int }}$ reads

$$
\mathcal{A}_{\text {int }}^{(2)}=\mathcal{A}_{\mathrm{int}}^{(2, \mathrm{~s})}+\mathcal{A}_{\mathrm{int}}^{(2, \mathrm{t})},
$$

with

$$
\begin{aligned}
\mathcal{A}_{\mathrm{int}}^{(2, \mathrm{~s})}= & \frac{\pi T}{4} K^{(2, \mathrm{~s})} \int d \mathbf{x} \sum_{r=0,3}(-1)^{r} \sum_{n_{1}, n_{2}, m} \sum_{\alpha} \\
& \times\left[\operatorname{tr}\left(\left(\tau_{r} \otimes s_{0}\right) \hat{Q}_{n_{1}, n_{1}+m}^{\alpha \alpha}(\mathbf{x})\right)\right] \\
& \times\left[\operatorname{tr}\left(\left(\tau_{r} \otimes s_{0}\right) \hat{Q}_{n_{2}+m, n_{2}}^{\alpha \alpha}(\mathbf{x})\right)\right],(3.6 \\
\mathcal{A}_{\mathrm{int}}^{(2, \mathrm{t})}= & \frac{\pi T}{4} K^{(2, \mathrm{t})} \int d \mathbf{x} \sum_{r=0,3}(-1)^{r} \sum_{n_{1}, n_{2}, m} \sum_{\alpha} \sum_{i=1}^{3} \\
& \times\left[\operatorname{tr}\left(\left(\tau_{r} \otimes s_{i}\right) \hat{Q}_{n_{1}, n_{1}+m}^{\alpha \alpha}(\mathbf{x})\right)\right] \\
& \times\left[\operatorname{tr}\left(\left(\tau_{r} \otimes s_{i}\right) \hat{Q}_{n_{2}+m, n_{2}}^{\alpha \alpha}(\mathbf{x})\right)\right],
\end{aligned}
$$

Here $K^{(2, \mathrm{~s})}$ and $K^{(2, \mathrm{~s})}$ are the spin-singlet and spintriplet two-body interaction amplitudes, respectively. In general, there also is an interaction amplitude in the particle-particle channel, which we neglect here.

Finally, for explicit calculations it is convenient to eliminate the constraints given by Eq. 3.1b. This can be done by means of the block matrix parametrization

$$
\hat{Q}=\left(\begin{array}{cc}
\sqrt{1-q q^{\dagger}} & q \\
q^{\dagger} & -\sqrt{1-q^{\dagger} q}
\end{array}\right) .
$$


Here the four block matrices represent, clockwise from upper left, the matrix elements of $\hat{Q}$ with frequency labels $n_{1}, n_{2}>0, n_{1}>0, n_{2}<0, n_{1}, n_{2}<0$, and $n_{1}<0, n_{2}>$ 0 .

From Eq. (3.4) it follows that a point-like, instantaneous three-body interaction term involving three number density fluctuations would take the form

$$
\begin{aligned}
\mathcal{A}_{\mathrm{int}}^{(3)} & =\frac{\pi^{2} T^{2}}{24} K^{(3, s)} \int d \boldsymbol{x} \sum_{r, s, t=0,3}(\sqrt{-1})^{r+s+t} \sum_{\substack{n_{1}, n_{2}, n_{3} \\
n_{4}, n_{5}, n_{6}}} \\
& \times \delta_{n_{1}+n_{3}+n_{5}, n_{2}+n_{4}+n_{6}} \sum_{\alpha}\left[\operatorname{tr}\left(\tau_{r} \otimes s_{0}\right) Q_{n_{1} n_{2}}^{\alpha \alpha}(\boldsymbol{x})\right] \\
& \times\left[\operatorname{tr}\left(\tau_{s} \otimes s_{0}\right) Q_{n_{3} n_{4}}^{\alpha \alpha}(\boldsymbol{x})\right]\left[\operatorname{tr}\left(\tau_{t} \otimes s_{0}\right) Q_{n_{5} n_{6}}^{\alpha \alpha}(\boldsymbol{x})\right] .
\end{aligned}
$$

We will now show that such a term is indeed produced by renormalizing the bare action $\mathcal{A}$ given in Eq. (3.1a).

\section{B. Loop expansion}

To proceed, we expand the action $\mathcal{A}$, Eq. 3.1a), in powers of $q$. To Gaussian order we obtain a quadratic form whose inverse determines the Gaussian propagators. In Fourier space, the latter read

$$
\left\langle{ }_{r}^{i} q_{12}\left(\boldsymbol{p}_{1}\right){ }_{s}^{j} q_{34}\left(\mathbf{p}_{2}\right)\right\rangle=\frac{G}{8} \delta_{r s} \delta_{i j}^{i} M_{r}^{-1} M_{12,34}\left(\boldsymbol{p}_{1}\right),
$$

with

$$
\begin{aligned}
& { }_{0,3}^{i} M_{12,34}^{-1}(\boldsymbol{p})=\delta_{1-2,3-4}\left[\delta_{13} \mathcal{D}_{n_{1}-n_{2}}(\boldsymbol{p})+\frac{\delta_{\alpha_{1} \alpha_{2}}}{n_{1}-n_{2}}\right. \\
& \left.\times\left(\mathcal{D}_{n_{1}-n_{2}}^{\nu_{i}}(\boldsymbol{p})-\mathcal{D}_{n_{1}-n_{2}}(\boldsymbol{p})\right)\right], \\
& { }_{1,2}^{i} M_{12,34}^{-1}(\boldsymbol{p})=-\delta_{13} \delta_{24} \mathcal{D}_{n_{1}-n_{2}}(\boldsymbol{p}) \text {. }
\end{aligned}
$$

Here $\nu_{0}=\mathrm{s}, \nu_{1,2,3}=\mathrm{t}$, and we have introduced the propagators

$$
\begin{aligned}
& \mathcal{D}_{n}(\boldsymbol{p})=1 /\left(\boldsymbol{p}^{2}+G H \Omega_{n}\right) \\
& \mathcal{D}_{n}^{\mathrm{s}}(\boldsymbol{p})=1 /\left(\boldsymbol{p}^{2}+G\left(H+K^{(2, s)}\right) \Omega_{n}\right), \\
& \mathcal{D}_{n}^{\mathrm{t}}(\boldsymbol{p})=1 /\left(\boldsymbol{p}^{2}+G\left(H+K^{(2, t)}\right) \Omega_{n}\right),
\end{aligned}
$$

which are proportional to the basic diffusion propagator defined in Eq. (2.2).

We now perform a systematic loop expansion, and concentrate on the renormalizations of the interaction terms. For simplicity, we consider only the particle-hole channel degrees of freedom, i.e., we neglect the propagators with $r=1,2$ above. A physical situation that realizes this approximation is, e.g., a system with magnetic impurities, which give the particle-particle channel propagators a mass, so they drop out of the soft-mode effective theory $\underline{6.8}$

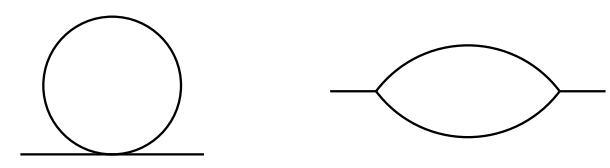

FIG. 3: Diagrams that renormalize the two-body interaction.

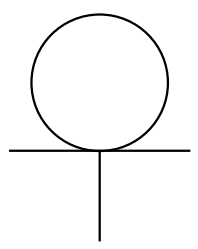

(a)

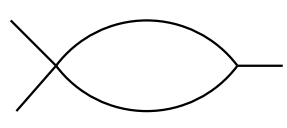

(b)

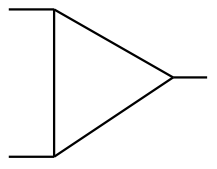

(c)
FIG. 4: Diagrams that can generate an effective three-body interaction.

At one-loop order, the two-body interactions get renormalized, and they acquire frequency and momentum dependences in the process. This effect is due to the diagrams shown in Fig. 3] and it has been studied in detail before ${ }^{6}$ However, there also are three-body interactions generated in the process. Let us concentrate on the pure spin-singlet term given by Eq. (3.8). To lowest order in powers of $q$, it will manifest itself in particular in a term

$$
\begin{aligned}
& (4 \pi T)^{2} \sum_{\substack{n_{1}, n_{2}, n_{3} \\
n_{4}, n_{5}, n_{6}}} \delta_{n_{1}+n_{3}-n_{5}, n_{2}+n_{4}-n_{6}} \int d \boldsymbol{x} d \boldsymbol{y} d \boldsymbol{z} \\
& \times \tilde{K}_{n_{1} n_{2} n_{3}, n_{4} n_{5} n_{6}}^{(3, s)}(\boldsymbol{x}, \boldsymbol{y}, \boldsymbol{z}){ }_{0}^{0} q_{n_{1} n_{2}}^{\alpha \alpha}(\boldsymbol{x}){ }_{0}^{0} q_{n_{3} n_{4}}^{\alpha \alpha}(\boldsymbol{y}){ }_{0}^{0} q_{n_{5} n_{6}}^{\alpha \alpha}(\boldsymbol{z}) .
\end{aligned}
$$

Here we have allowed for a frequency and real-space dependence of the three-body interaction amplitude, and for simplicity we only consider the ${ }_{0}^{0} q$ components of the matrices $q$. A vertex with the structure of Eq. (3.10) can in principle be generated by any of the three diagrams shown in Fig. 4

It is easy to see that diagram (a) in Fig. 目 does not contribute to a three-body interaction; the realizations of this diagram that have the correct replica structure do not have a frequency structure consistent with Eq. (3.10). This is consistent with our conclusion, in Sec. III that there are no contributions to $K^{(3)}$ that are linear in $K^{(2)}$. Of the remaining two diagrams, (c) is at least of cubic order in $K^{(2)}$, and in particular produces terms that have the structure of $K^{(3,1)}$ in Eq. (2.4a). Diagram (b) has contributions of the correct structure that are of $O\left(\left(K^{(2)}\right)^{2}\right)$, as well as contributions of higher order. The easiest check for the existence of $K^{(3)}$ therefore consists of a systematic calculation of diagram (b), keeping only terms of second order in the two-body interaction amplitude $K^{(2, s)}$. Such a calculation yields 


$$
\tilde{K}_{123,456}^{(3, s)}(\boldsymbol{x}, \boldsymbol{y}, \boldsymbol{z})=K_{123,456}^{(3, s)}(\boldsymbol{x}, \boldsymbol{y}, \boldsymbol{z})+K_{341,256}^{(3, s)}(\boldsymbol{y}, \boldsymbol{x}, \boldsymbol{z})
$$

where $K^{(3, s)}$ reads, in Fourier space,

$$
\begin{aligned}
& K_{123,456}\left(\mathbf{k}_{1}, \mathbf{k}_{2}, \mathbf{k}_{3}\right)=\left(G K_{s} / 8\right)^{2} \int_{\mathbf{p}} \\
& \times \\
& \times-2 \Theta(5 \geq 1-4)\left[\left(\mathbf{p}+\mathbf{k}_{1}\right)^{2}+\left(\mathbf{p}+\mathbf{k}_{2}\right)^{2}+G H \Omega_{1-2}+G H \Omega_{3-2}\right]\left(\mathcal{D}_{3-2}(\mathbf{p})\right)^{2} \mathcal{D}_{1-4}\left(\mathbf{p}-\mathbf{k}_{3}\right) \\
&+2 \Theta(5<1-4)\left[\left(\mathbf{p}+\mathbf{k}_{1}\right)^{2}+\left(\mathbf{p}+\mathbf{k}_{2}\right)^{2}+G H \Omega_{1-4}+G H \Omega_{3-4}\right]\left(\mathcal{D}_{1-4}(\mathbf{p})\right)^{2} \mathcal{D}_{3-2}\left(\mathbf{p}-\mathbf{k}_{3}\right) \\
&+2 \Theta(5>1+3)\left[\mathcal{D}_{5-1}(\mathbf{p}) \mathcal{D}_{1-6}\left(\mathbf{p}-\mathbf{k}_{3}\right)+\mathcal{D}_{5-3}(\mathbf{p}) \mathcal{D}_{5-4}\left(\mathbf{p}-\mathbf{k}_{2}\right)\right] \\
&-2 \Theta(5 \leq 1+3)\left[\mathcal{D}_{5-2}(\mathbf{p}) \mathcal{D}_{2-6}\left(\mathbf{p}-\mathbf{k}_{3}\right)+\mathcal{D}_{3-6}(\mathbf{p}) \mathcal{D}_{4-6}\left(\mathbf{p}-\mathbf{k}_{2}\right)\right] \\
&-2 \Theta(5 \geq 3-2)\left[\mathcal{D}_{5-3}(\mathbf{p}) \mathcal{D}_{3-6}\left(\mathbf{p}-\mathbf{k}_{3}\right)+\mathcal{D}_{5-3}(\mathbf{p}) \mathcal{D}_{5-4}\left(\mathbf{p}-\mathbf{k}_{2}\right)\right] \\
&+2 \Theta(5<3-2)\left[\mathcal{D}_{5-4}(\mathbf{p}) \mathcal{D}_{4-6}\left(\mathbf{p}-\mathbf{k}_{3}\right)+\mathcal{D}_{3-6}(\mathbf{p}) \mathcal{D}_{4-6}\left(\mathbf{p}-\mathbf{k}_{2}\right)\right] \\
&-2 \Theta(5 \geq 1-2) \mathcal{D}_{5-4}(\mathbf{p}) \mathcal{D}_{5-4-1+2}\left(\mathbf{p}-\mathbf{k}_{1}\right)+2 \Theta(5<1-2) \mathcal{D}_{1-6}(\mathbf{p}) \mathcal{D}_{1-6-3+4}\left(\mathbf{p}-\mathbf{k}_{2}\right) \\
&\left.+2 \mathcal{D}_{5-4}(\mathbf{p}) \mathcal{D}_{5-4+1-2}\left(\mathbf{p}+\mathbf{k}_{1}\right)-2 \mathcal{D}_{1-6}(\mathbf{p}) \mathcal{D}_{1-6+3-4}\left(\mathbf{p}+\mathbf{k}_{2}\right)\right\}
\end{aligned}
$$

Here $\int_{\boldsymbol{p}}=\int d \boldsymbol{p} /(2 \pi)^{d}, 1 \equiv n_{1}$, etc., and the symbols $\Theta(5 \geq 1-4) \equiv \Theta\left(n_{5}-n_{1}+n_{4}\right)$, etc., with the second $\Theta$ denoting the usual Heavyside step function, express constraints among the frequencies.

This result demonstrates that a term with the structure of $K^{(3,2)}$, Eq. (2.5), gets indeed generated upon renormalization of an action with a pure two-body interaction. In addition, there exist terms that represent $K^{(3,1)}$, Eq. (2.4a), as well as spin-triplet and mixed singlet-triplet three-body interactions. It is also plausible that four- and higher-body interaction terms are generated by the same mechanism, and the existence of particular diagrams with the appropriate structure is easily verified.

We note that the momentum integral in Eq. (3.11b) diverges for small external wavevectors $\boldsymbol{k}$, or small external frequencies $\Omega$, as $1 / \boldsymbol{k}^{2}$ or $1 / \Omega$, in agreement with the remark after Eq. 2.4b). The RG interpretation of this divergence is given in the next section.

\section{PHYSICAL EFFECTS DUE TO EFFECTIVE MANY-BODY INTERACTIONS}

\section{A. Structure of renormalization-group flow equations}

Since structurally new terms have appeared in our action under renormalization, we need to add these terms to the action and start the renormalization process over again. The action as given by Eqs. (3.113.6) thus must be augmented by Eq. (3.8) 20 The renormalization of this action then proceeds along standard lines. The result is obviously a generalization of the known flow equations for the model with two-particle interactions only. For our present purposes we are interested only in the general structure of these flow equations, which can be obtained without a detailed calculation.

We choose the scale dimension of a length $L$ to be $[L]=1$, and that of imaginary time $\tau$ to be $[\tau]=d$ in $d$ spatial dimensions ${ }^{21}$ The field $q(\boldsymbol{x})$ we choose to be dimensionless. The bare scale dimension of $G$ is then $d-2 \equiv \epsilon$, the bare scale dimensions of $H, K^{(2, \mathrm{~s})}$ and $K^{(2, \mathrm{t})}$ are zero. The bare scale dimension of $K^{(3)}$ is $-d$, due to the extra factor of $T$ that appears in the threebody interaction term, Eq. (3.8), compared to the twobody interaction. If we denote the renormalized, scale dependent counterparts of these coupling constants by $g$, $h, k_{\mathrm{s}}, k_{\mathrm{t}}$, and $k_{3}$, respectively, we thus have to zero-loop order

$$
\begin{aligned}
\frac{d g}{d \ell} & =-\epsilon g \\
\frac{d h}{d \ell} & =\frac{d k_{\mathrm{s}}}{d \ell}=\frac{d k_{\mathrm{t}}}{d \ell}=0 \\
\frac{d k_{3}}{d \ell} & =-(2+\epsilon) k_{3}
\end{aligned}
$$

Here $\ell=\ln b$, with $b$ the renormalization-group length rescaling factor.

To find the higher-loop order terms explicitly requires a detailed calculation. For $k_{3}=0$, the result is known completely to one-loop order, and selectively to two-loop order $\stackrel{6}{\underline{6}}$ For all universality classes, the structure is,

$$
\begin{aligned}
\frac{d g}{d \ell} & =-\epsilon g+g^{2} f_{g}^{(1)}\left(\gamma_{\mathrm{s}}, \gamma_{\mathrm{t}}\right)+g^{3} f_{g}^{(2)}\left(\gamma_{\mathrm{s}}, \gamma_{\mathrm{t}}\right) \\
\frac{d h}{d \ell} & =h g f_{h}^{(1)}\left(\gamma_{\mathrm{s}}, \gamma_{\mathrm{t}}\right)+h g^{2} f_{h}^{(2)}\left(\gamma_{\mathrm{s}}, \gamma_{\mathrm{t}}\right) \\
\frac{d \gamma_{\mathrm{s}}}{d \ell} & =g f_{\mathrm{s}}^{(1)}\left(\gamma_{\mathrm{s}}, \gamma_{\mathrm{t}}\right)+g^{2} f_{\mathrm{s}}^{(2)}\left(\gamma_{\mathrm{s}}, \gamma_{\mathrm{t}}\right) \\
\frac{d \gamma_{\mathrm{t}}}{d \ell} & =g f_{\mathrm{t}}^{(1)}\left(\gamma_{\mathrm{s}}, \gamma_{\mathrm{t}}\right)+g^{2} f_{\mathrm{t}}^{(2)}\left(\gamma_{\mathrm{s}}, \gamma_{\mathrm{t}}\right)
\end{aligned}
$$


where $\gamma_{\mathrm{s}, \mathrm{t}}=k_{\mathrm{s}, \mathrm{t}} / h$. We note that $-1 \leq \gamma_{\mathrm{s}}<0$, and $\gamma_{\mathrm{t}}>0$, and the various functions $f$ are well-behaved in the limit $\gamma_{\mathrm{s}, \mathrm{t}} \rightarrow 0 \underline{\underline{6}}$

In the presence of $k_{3}$, we need to consider, first, the influence of $k_{3}$ on the flow of the other coupling constants, and, second, the $k_{3}$-flow equation itself. Simple counting arguments show that $k_{3}$ cannot produce singular (in $d=2$ ) one-loop renormalizations of the other coupling constants. For instance, consider the second diagram in Fig. [3 with one of the vertices replaced by a three-body interaction. Due to the additional factor of $T$ in Eq. (3.8) compared to Eqs. (3.6) this diagram will have an extra frequency integration compared to the diagram with both vertices given by two-body interactions, and will thus not be infrared singular. The structure of the flow equation for $k_{3}$ itself is therefore more important than the modifications of Eqs. 4.2a-4.2d, and the crucial question is whether is it possible to overcome the negative bare scale dimension of $k_{3}$. The most interesting term is therefore the one-loop renormalization of $k_{3}$ that is proportional to $k_{3}$ itself. Such terms exist; they are realized, e.g., by diagrams (b) and (c) in Fig. 4 with one of the three-point vertices replaced by a three-body interaction. Simple counting arguments show that the structure of the $k_{3}$-flow equation to one-loop order is

$$
\frac{d k_{3}}{d \ell}=-(2+\epsilon) k_{3}+k_{3} g f_{3}^{(1)}\left(\gamma_{\mathrm{s}}, \gamma_{\mathrm{t}}\right)+g^{2} \tilde{f}_{3}^{(1)}\left(\gamma_{\mathrm{s}}, \gamma_{\mathrm{t}}, h\right) \text {. }
$$

Note that the last term on the right-hand side of Eq. (4.2e) is independent of $k_{3}$. It represents the contributions that generate $k_{3}$ in the first place, for instance, the one given by Eqs. (3.11).

In general, adding a new $R G$ variable to a set of flow equations can have any one of three effects by virtue of the new eigenvalue it adds to the set of equations linearized about any fixed point. First, it may be truly irrelevant in the sense that it does not qualitatively change any aspects of the RG flow in its absence. Second, it may be irrelevant with respect to a fixed point that exists in its absence, but change the flow outside of the basin of attraction of this fixed point. (It will in general also change the size of this basin of attraction.) Third, it may be relevant with respect to the original fixed point. In the latter two cases, outside of the basin of attraction of the original fixed point, if any, it may either lead to a new fixed point, or to flow towards strong coupling. With this in mind, we next discuss possible types of fixed points of the above flow equations.

\section{B. Weak-coupling fixed points}

In the usual perturbative RG treatment one looks for fixed points of the flow equations, Eqs. (4.2), where $g$ is small of $O(\epsilon)$, and $\gamma_{\mathrm{s}}$ and $\gamma_{\mathrm{t}}$ are at most of $O(1)$. This is our definition of a weak-coupling fixed point ${ }^{22}$ It follows from Eq. 4.2e that the new scaling operator introduced by the presence of $k_{3}$ will have a scale dimension of $-2+O(\epsilon)$ with respect to such a fixed point. This is assured by the bare scale dimension of $k_{3},\left[k_{3}\right]=-d$, which cannot be overcome by the small one-loop term. In this context it is important to mention that $k_{3}$ itself does have a component that is marginal in $d=2$. This follows from the fact that the one-loop term in Eq. (4.2e) has a contribution that is independent of $k_{3}$, see the remark after that equation, and Eqs. (3.11). However, this component just reflects the scaling behavior of the other coupling constants, $g, h, k_{\mathrm{s}}$, and $k_{\mathrm{t}}$, and it does not lead to a new eigenvalue of the linearized RG flow equations. In other words, $k_{3}$ is not a proper scaling operator, and the scaling operator related to $k_{3}$ has the components that are marginal in $d=2$ projected out. An analogous phenomenon in $\phi^{4}$-theory is discussed in the Appendix.

We also note that, alternatively, one could treat the three-body interaction generated by Eqs. (3.11) as a truly long-ranged interaction with a bare scale dimension of $-2 \epsilon$. Such a procedure would lead to the same conclusion, since the part that is marginal in $d=2$ would just reflect the scaling behavior of the two-body interaction amplitudes. The one-loop term independent of $k_{3}$ thus reflects the long-range nature of the RG-generated threebody interaction; see also the remark at the end of Sec. III This observation justifies our using a point-like threebody interaction amplitude despite the fact that the one generated in perturbation theory was long-ranged. In the Appendix we discuss a similar feature of $\phi^{4}$-theory.

We conclude that the many-body interactions are indeed important for weak-coupling fixed points, as one would have expected. However, since they do not lead to new marginal (in $d=2$ ) scaling operators, the relevant physics is already contained in the renormalization of the two-body interaction constants. Weak-coupling fixed points will thus always be perturbatively stable with respect to $k_{3}$, and also with respect to higher many-body interactions, i.e., they have a finite basin of attraction. This in turn implies that the coupling of $k_{3}$ to the other coupling constants cannot change the critical behavior, it will merely lead to power-law corrections to scaling. In particular, all of the perturbative metal-insulator transition fixed points that are known to exist for the generalized nonlinear sigma-model defined in Sec. III are stable with respect to $k_{3}$.

\section{Strong-coupling fixed points}

Let us now consider strong-coupling fixed points, where the ratio of successive terms in the loop expansion is not necessarily some power of $\epsilon$. This can happen if the fixed point value of $\gamma_{\mathrm{t}}$ is large, of $O(1 / \epsilon)$, or infinite, even if $g$ is still of $O(\epsilon)$. Of course, another possibility is that $g=O(1)$. No controlled theories exist of metal-insulator transitions that are governed by such fixed points, but they are structurally clearly possible. Explicit, if uncontrolled, examples of fixed points where both $g$ and an interaction coupling constant are of $O(1)$ 
have been given in Refs. 10 and 11.23 It is also believed that strong-coupling physics governs the behavior in certain $2-d$ sytems, where the interaction strength may be the dominant energy scale in the problem 12

The arguments in the previous subsection that ensure the irrelevance of $k_{3}$ obviously break down for such strong-coupling fixed points. We stress that this may be true even if the fixed-point value of $g$ is still small. The point is that with, e.g., $g=O(\epsilon)$ and $\gamma_{\mathrm{t}}=O(1 / \epsilon)$, $g \gamma_{\mathrm{t}}=O(1)$, and hence the one-loop term in Eq. (4.2e) can overwhelm the bare scale dimension of $k_{3}$. The same arguments hold for the higher many-body interaction terms, although to a lesser degree, since for them a larger negative bare scale dimension must be overcome in order to make them relevant.

We conclude that the many-body interaction terms cannot be dismissed a priori in any strong-coupling regime, where the dimensionless interaction amplitudes are large, even if the disorder is still small. In particular, they are likely to play a role in the resolution of the two-dimensional metal-insulator transition problem.

\section{SUMMARY, AND CONCLUSION}

To summarize, we have shown that many-body interactions are generated under renormalization of an action for interacting disordered electrons that contains two-body interactions only. Such interactions turn out to be irrelevant with respect to the perturbative fixed points that describe metal-insulator transitions in $d=2+\epsilon$. However, they need to be examinated, and they likely contribute to the leading behavior, in any strong-coupling theory. This implies in particular that even if strongcoupling solutions for the generalized nonlinear sigmamodel with two-body interactions could be found, such solutions would be incomplete, and probably physically wrong. The task of determining the strong-coupling behavior of such systems, and in particular the situation in $d=2$, is thus even harder than previously assumed.

We conclude by recapitulating two aspects of our technical development that can easily lead to confusion. First of all, let us come back to the infrared divergence of the integral in Eq. (3.11b). Naively, this infrared divergence seems to offset the extra factor of temperature compared to the two-body interaction, making the three-body interaction marginal by power counting in $d=2$. The same argument applies to higher many-body interaction amplitudes, which carry additional factors of temperature, but come with even more divergent loop integrals. As we show explicitly by analyzing an analogous effect in $\phi^{4}$-theory in the appendix, this simple argument is fallacious and the many-body interactions are perturbatively irrelevant, but they are likely to play an important role in nonperturbative regimes. Second, we have focussed on one particular three-body interaction term, namely, a spin-singlet three-body interaction. For this term, we have calculated all contributions to second order in the spin-singlet two-body interaction within a well-defined model. This proves the existence of many-body interactions in effective field theories for electrons, but our calculation is sensitive to only a small subclass of such terms. Many-body interactions coupling four and more electrons, spin-triplet interactions, and terms coupling singlet and triplet density fluctuations certainly exist, and they all need to be examined in order to systematically deal with strong-coupling effects.

Finally, we note that existing theories of the AndersonMott transition seem to lead to the conclusion that it is very similar to an Anderson transition ${ }^{24}$ However, it is reasonable to assume that in strongly correlated systems the metal-insulator transition should more closely resemble a Mott transition 13 This suggests the existence of a saddle in parameter space separating the fixed points that describe the two transitions. The disordered Mott transition is likely described by a strong-coupling fixed point. As discussed after Eqs. [4.2), on the strongcoupling side of such a saddle the many-particle interactions are likely to be important, even if the weak-coupling Anderson or Anderson-Mott fixed point is locally stable with respect to them. In this context it is interesting to note that a Landau theory for the Anderson-Mott transition in high dimensions $(d>6)^{14.15}$ found indeed that for weak interactions, an Anderson transition takes place with increasing disorder, while for strong interactions the metal-insulator transition has a different nature. It is likely that the many-body interactions discussed in this paper are important for understanding the missing link between this theory in high dimensions, and the usual treatment of the metal-insulator transition problem near two-dimensions.

\section{Acknowledgments}

We would like to thank John Toner for an enlightening discussion. This work was supported by the NSF under grant Nos. DMR-01-32555, and DMR-01-32726.

\section{APPENDIX A: GENERATION OF HIGHER-ORDER TERMS IN $\phi^{4}$-THEORY}

In this appendix we recall some aspects of $\phi^{4}$-theory that are analogous to the generation of many-body interactions discussed in the main part of the paper. See Refs. 161718 for a derivation of the results summarized below.

Consider scalar $\phi^{4}$-theory, with an action

$S[\phi]=-\frac{1}{2} \int d \boldsymbol{x} \phi(\boldsymbol{x})\left[r-\nabla^{2}\right] \phi(\boldsymbol{x})-\frac{u_{4}}{4} \int d \boldsymbol{x} \phi^{4}(\boldsymbol{x})$.

Upon renormalization, a $\phi^{6}$-term with a coupling constant $u_{6}$ is generated by means of the diagram shown in Fig. [5] This diagram represents a momentum inte- 


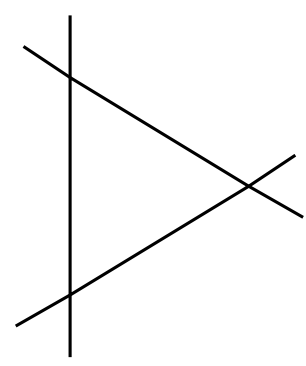

FIG. 5: Diagram that generates a $\phi^{6}$-term from $\phi^{4}$-terms.

gral over three propagators. In perturbation theory, and at criticality, this integral is strongly infrared divergent. Naively, this raises the question whether $u_{6}$ can really be irrelevant with respect to the Wilson-Fisher fixed point. To investigate this, we consider the RG flow equations. Adding a $\phi^{6}$-term to the action, one easily finds, within a momentum-shell RG, and to one-loop order,

$$
\begin{aligned}
\frac{d r}{d \ell} & =2 r+\frac{3 u_{4}}{1+r} \\
\frac{d u_{4}}{d \ell} & =\epsilon u_{4}-\frac{9 u_{4}^{2}}{(1+r)^{2}}+\frac{10 u_{6}}{1+r} \\
\frac{d u_{6}}{d \ell} & =-2(1-\epsilon) u_{6}-\frac{45 u_{4} u_{6}}{(1+r)^{2}}+\frac{27 u_{4}^{3}}{(1+r)^{3}}
\end{aligned}
$$

where $\epsilon=4-d$. Linearization of these flow equations about the Wilson-Fisher fixed point $\left(r^{*}, u_{4}^{*}, u_{6}^{*}\right)=$ $\left(-\epsilon / 6+O\left(\epsilon^{2}\right), \epsilon / 9+O\left(\epsilon^{2}\right), \epsilon^{3} / 54+O\left(\epsilon^{4}\right)\right)$ yields three RG eigenvalues

$$
\begin{aligned}
& \lambda_{1}=2-\epsilon / 3+O\left(\epsilon^{2}\right) \\
& \lambda_{2}=-\epsilon+O\left(\epsilon^{2}\right) \\
& \lambda_{3}=-2-3 \epsilon+O\left(\epsilon^{2}\right)
\end{aligned}
$$

$\lambda_{1}=1 / \nu>0$ is the inverse correlation length exponent, $\lambda_{2}$ is the scale dimension of the least irrelevant operator, and $\lambda_{3}$ is irrelevant even for $\epsilon=0$. The infrared properties of the triangle diagram thus do not lead to another (besides $\lambda_{2}$ ) eigenvalue of $O(\epsilon)$. This is true even though it does lead to a leading scaling behavior of $u_{6}$ that is given by $u_{6}(b \rightarrow \infty) \sim b^{-\epsilon}$, as can be seen by solving the flow equations. However, this just means that $u_{6}$ is not a proper scaling operator, since it couples to $u_{4}$. With $\delta u_{4}=u_{4}-u_{4}^{*}$ and $\delta u_{6}=u_{6}-u_{6}^{*}$, the proper next-to-least irrelevant scaling operator is $g_{6}=\delta u_{6}-\left(\epsilon^{2} / 2\right) \delta u_{4}+O\left(\epsilon^{3}\right)$. Its scale dimension is $\left[g_{6}\right]=\lambda_{3}$, so $g_{6}$ is indeed irrelevant with scale dimension -2 in $d=4$.

This phenomemon of the generation of a new operator that is irrelevant with respect to the perturbative fixed point in $d=4-\epsilon$ is completely analogous to the irrelevance of the three-body interaction with respect to the perturbative metal-insulator transition fixed points in $d=2+\epsilon$, and the analogy extends to the long-rangedness of the new interaction. It is interesting to note, however, that there is no general $a$ priori guarantee that $g_{6}$ will no be relevant in, say, $d=3$. Indeed, the bare scale dimension of $u_{6}$ is $-2+2 \epsilon$, which naively implies a marginal operator in $d=3$. The one-loop correction switches the sign of the $O(\epsilon)$ correction, see Eq. (A3C), so the oneloop approximation to $\lambda_{6}$ makes $g_{6}$ more irrelevant with increasing $\epsilon$, not less. However, it is important to keep in mind that, (1) this change of sign is a special property of $\phi^{4}$-theory, which can be seen only by means of an explicit calculation, and, (2) there is no guarantee that high-order terms in the $\epsilon$-expansion will not have a positive sign and make $\lambda_{3}$ positive in $d=3$. In $\phi^{4}$-theory there are no indications that this is the case, but it could happen in a different, more complicated field theory.
1 See, e.g., J. Zinn-Justin, Quantum Field Theory and Critical Phenomena (Oxford University Press, Oxford, 1996).

2 C. Itzykson and J.-B. Zuber, Quantum Field Theory (McGraw-Hill, New York, 1980), ch. 11-3.

3 S. Weinberg, The Quantum Theory of Fields, vol. II (Cambridge University Press, Cambridge, 1996), ch. 21.3.

${ }^{4}$ E. H. Hauge, in Transport Phenomena, Lecture Notes in Physics No. 31, edited by G. Kirczenow and J. Marro (Springer, New York, 1974), p. 337.

5 K. I. Wysokiński, D. Belitz, and T. R. Kirkpatrick, Phys. Rev. E 52, 612 (1995).

${ }^{6}$ For a review, see, D. Belitz and T. R. Kirkpatrick, Rev. Mod. Phys. 66, 261 (1994).

7 A. M. Finkelstein, Zh. Eksp. Teor. Fiz. 84, 168 (1983), [Sov. Phys. JETP 57, 97 (1983)].

8 A. M. Finkelstein, Zh. Eksp. Teor. Fiz. 86, 367 (1984), [Sov. Phys. JETP 59, 212 (1984)].

9 C. Castellani, C. D. Castro, P. A. Lee, and M. Ma, Phys. Rev. B 30, 572 (1984).

10 T. R. Kirkpatrick and D. Belitz, J. Phys. Cond. Matt. 4,
L37 (1992).

11 W. L. McMillan, Phys. Rev. B 24, 2739 (1981).

12 E. Abrahams, S. V. Kravchenko, and M. P. Sarachik, Rev. Mod. Phys. 73, 251 (2001).

13 N. F. Mott, Metal-Insulator Transitions (Taylor \& Francis, London, 1990).

14 T. R. Kirkpatrick and D. Belitz, Phys. Rev. Lett. 73, 862 (1994).

15 D. Belitz and T. R. Kirkpatrick, Z. Phys. B 98, 513 (1995).

16 S.-K. Ma, Modern Theory of Critical Phenomena (Benjamin, Reading, MA, 1976).

17 K. G. Wilson and J. Kogut, Phys. Rep. 12, 75 (1974).

18 J. Cardy, Scaling and Renormalization in Statistical Physics (Cambridge University Press, Cambridge, 1996).

19 What constitutes "weak" or "strong" interactions or disorder depends, inter alia, on the dimensionality of the system, and no general criteria can be given. See Sec. [V] for a discussion that elaborates on this remark.

20 Adding a term with a point-like three-body interaction amplitude is sufficient, even though the interaction generated 
in perturbation theory is long-ranged. See Sec. IVB and the Appendix for a justification.

21 Within the spirit of standard phase transition theory, ${ }^{16}$ one would choose $H$ in Eq. 3.1a to be marginal, which would make $[\tau]=z$, with $z$ the dynamical exponent. However, traditionally the scale dimension $[\tau]$ of the imaginary time has been fixed in descriptions of the Anderson-Mott transition, with the scale dimension of $H$ making up for the difference between $[\tau]$ and $z{ }^{\underline{6}}$ The choice $[\tau]=d$ is motivated by the fact that $[\tau]=z=d$ at the Anderson metal-insulator transition of noninteracting electrons, and that for all known universality classes of the AndersonMott metal-insulator transition of interacting electron in $d=2+\epsilon,[\tau]=d+O(\epsilon)$.

${ }^{22}$ More generally, at a weak-coupling fixed point successive terms in the loop expansion of the flow equations become smaller by some power of $\epsilon$. It is conceivable that this condition might be violated for $\gamma_{\mathrm{s}} \rightarrow-1$, e.g., by powers of $1 /\left(1+\gamma_{\mathrm{s}}\right)$ occuring at some order in the loop expansion, although no examples of such behavior are known.

23 AlthoughMcMillan's paper, Ref. 11, contained a technical mistake, this should not distract from the attractiveness of the structure of his scaling theory.

24 Even though there are qualitative differences between an Anderson-Mott transition and an Anderson transition, e.g., the former has a critical density of states, this statement is true in the following sense: Both transitions are driven by diffusive modes, and the lower critical dimensionality is equal to 2 . The presence of the interaction just modifies the diffusive modes. A Mott transition, by contrast, with or without disorder, is fundamentally driven by interactions. 